\title{
A Cross-Cultural Study of Physician Treatment Decisions for Demented Nursing Home Patients Who Develop Pneumonia
}

\author{
Margaret R. Helton, $M D^{1}$ \\ Jenny T. van der Steen, $P b D^{2}$ \\ Timotby P. Daaleman, DO, MPH ${ }^{1,3}$ \\ George R. Gamble, $P b D^{1}$ \\ Miel W. Ribbe, $M D, P b D^{2}$ \\ 'Department of Family Medicine, Univer- \\ sity of North Carolina, Chapel Hill, NC \\ ${ }^{2}$ Institute for Research in Extramural Medi- \\ cine (EMGO), and Departments of Nursing \\ Home Medicine and Public and Occupa- \\ tional Health, VU University Medical \\ Center, Amsterdam, the Netherlands \\ ${ }^{3}$ Program on Aging, Disability, and Long- \\ Term Care, Cecil G. Sheps Center for \\ Health Services Research, University of \\ North Carolina, Chapel Hill, NC
}

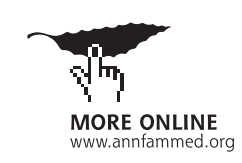

Conflicts of interest: none reported

\section{CORRESPONDING AUTHOR}

Margaret R. Helton, MD

Department of Family Medicine

CB \# 7595

University of North Carolina

Chapel Hill, NC 27599-7595

margaret_helton@med.unc.edu

\begin{abstract}
PURPOSE We wanted to explore factors that influence Dutch and US physician treatment decisions when nursing home patients with dementia become acutely ill with pneumonia.

METHODS Using a qualitative semistructured interview study design, we collected data from 12 physicians in the Netherlands and 12 physicians in North Carolina who care for nursing home patients. Our main outcome measures were perceptions of influential factors that determine physician treatment decisions regarding care of demented patients who develop pneumonia.

RESULTS Several themes emerged from the study. First, physicians viewed their patient care roles differently. Dutch physicians assumed active, primary responsibility for treatment decisions, whereas US physicians were more passive and deferential to family preferences, even in cases when they considered families' wishes for care as inappropriate. These family wishes were a second theme. US physicians reported a perceived sense of threat from families as influencing the decision to treat more aggressively, whereas Dutch physicians revealed a predisposition to treat based on what they perceived was in the best interest of the patient. The third theme was the process of decision making whereby Dutch physicians based decisions on an intimate knowledge of the patient, and American physicians reported limited knowledge of their nursing home patients as a result of lack of contact time.
\end{abstract}

CONCLUSION Physician-perceived care roles regarding treatment decisions are influenced by contextual differences in physician training and health care delivery in the United States and the Netherlands. These results are relevant to the debate about optimal care for patients with poor quality of life who lack decision-making capacity.

Ann Fam Med 2006;4:221-227. DOI: 10.1370/afm.536.

\section{INTRODUCTION}

$\mathrm{D}$ ementia-a progressive illness of cognitive, functional, and physical deterioration-is a major cause of morbidity and mortality. ${ }^{1}$

The sequelae of advanced dementia frequently lead to pneumonia, which can be a terminal event in long-term care settings. ${ }^{2,3}$ The development of pneumonia in elderly patients with dementia often raises multiple ethical and clinical issues for physicians, and there is wide variation in the care of these patients across cultures. For example, in Dutch long-term care settings, demented residents who develop pneumonia are rarely hospitalized but are treated empirically and symptomatically within the nursing home. ${ }^{4}$ In the United States, however, many patients acquiring pneumonia in long-term care facilities are transferred to acute-care hospitals. ${ }^{5-7}$ Often the result is aggressive care involving use of diagnostic interventions, intravenous antibiotics, and rehydration therapy ${ }^{7,8}$ despite evidence 
that treatment in a hospital rather than the nursing home does not improve outcomes, such as mortality ${ }^{9,10}$

The wide variation between US and Dutch approaches to this care has been attributed to contextual differences on 2 levels: the system of organizing nursing home care, and the legal and social understanding of end-of-life decision making. ${ }^{8}$ These contextual differences are frequently embedded in both professional and family values regarding end-oflife decision making. A qualitative study of US family members of nursing home residents with dementia, for example, found families had painful emotional needs that dominated decision making. ${ }^{11}$ In both cultures, however, physicians are still ultimately responsible and accountable for this care, because a physician's order is necessary for initiating and coordinating care between acute-care hospitals and long-term care. To understand the variation in this care further, we conducted an exploratory study to determine which factors Dutch and US physicians perceive as important in their treatment decisions regarding nursing home patients with dementia who become acutely ill with pneumonia.

\section{METHODS}

We used a qualitative research design with semistructured interviews conducted by the principal investigator $(\mathrm{MRH})$. Purposeful sampling in the United States and the Netherlands identified 2 sets of physicians who care for nursing home patients. In both countries, we sought physicians who represented a wide range of clinical experience, worked in both rural and urban settings, and varied in their care approaches at the end of life. We anticipated that 12 physicians in each country would be sufficient to reach a threshold level of data saturation. An interview guide (Supplemental Appen-

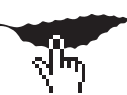

dix 1, available at http://www.annfammed.org/cgi/ content/full/4/3/221/DC1) was used to direct exploration of factors that potentially influence medical decision making, as well as physicians' thoughts and experiences in this area of clinical care. Participants were asked to describe and reflect on 2 actual cases of pneumonia in nursing home patients with dementia: one in which a curative or aggressive approach was undertaken, and one in which a palliative or comfort care approach was chosen.

The interviews were conducted in English, tape recorded, professionally transcribed, and checked for accuracy by the principal investigator, who is a family physician. Initial coding was performed by the principal (MRH) and coinvestigator (TPD), and the interviews were read to identify emerging patterns using editing analysis in which meaningful segments of text are coded to construct and interpret common themes. ${ }^{12}$
Concurrent independent analyses were performed by an American anthropologist with expertise in qualitative research (GRG) and a Dutch epidemiologist experienced in Dutch physician decision making and nursing home care (JTS). All investigators subsequently met and iteratively reviewed the codes and categories to reach consensus. Themes were compared within and across interviews, as well as within and across cultures. All data were analyzed using qualitative research software (QSR N6). ${ }^{13}$ The study was approved by the University of North Carolina Committee on the Protection of the Rights of Human Subjects.

\section{RESULTS}

Our participants all cared for patients with dementia who resided in nursing homes. The US physicians were board-certified in their specialty, 8 in family medicine and 4 in internal medicine, with 6 having additional training in geriatrics. The average age was 46 years (range 35 to 57 years); 4 were women. The Dutch physicians were trained in nursing home medicine, which is a separate specialty in the Netherlands, and had an average age of 44 years (range 33 to 60 years); 5 were women. Our analysis of the interviews resulted in the identification of 3 domains or thematic areas influencing physician decision making for demented nursing home patients who develop pneumonia. By the last few interviews, data saturation was achieved, namely, no new themes or ideas emerged.

\section{Physician Patient Care Role}

The physician's patient care role was an implicit and overt set of expectations and assumptions regarding the physician's diagnostic strategies and provision of care. US physicians described a passive knowledge of the patient typified by a reliance on technology, a proxy assessment by nursing home staff or emergency department physicians, and discontinuity. These physicians reported often not knowing their nursing home patients because of the infrequency of visits or lack of time or opportunity to get to know the patients and their families.

"My interactions with the family are hit or miss. As doctors, we sneak in and we sneak out. The nurses know them very well, but I don't have occasion to have that much interaction with them" (US physician 5).

"If you haven't talked to the family as much as you liked to, you might not be on the same page, and sometimes it seems safer to send them [the patients] to the ER and at least they get a good evaluation there with good x-rays, and then maybe the discussions can be made there" (US physician 6).

In contrast, Dutch physicians depicted a contextual knowledge of the patient encompassing a relationship 
of continuity, personal and first-hand understanding, and appreciation of the patient's quality of life and functional status.

"What I try to do in each patient is to carefully look at how he is, try to get an idea how he feels, how he functions in the ward, how he relates still to other people, how happy he or she is" (Dutch physician 10).

As a result of this knowledge, the Dutch physicians reported they seldom use chest radiographs, blood tests, or technology in their assessment; instead, they rely on clinical signs and symptoms and their physical examination. This reliance on clinical acumen by Dutch physicians was tied to a perceived duty to protect the patient from medical interventions.

"I feel very representative of medical society that has offered so many things that it is my duty to bring them out of the pace of the medical path we have come along until now, and to bring them more to human insight" (Dutch physician 12).

Although Dutch physicians highly value family input, they saw their role as assuming responsibility for the decision and were less willing to render care they saw as inappropriate, even if it is against family wishes.

"Before I make the decision, I want to know how the family thinks about the problem, or how the family thinks mother or father would think about this problem. I make it clear that they don't have to make the decision. I make the decisions. And I think they quite often see that as a relief" (Dutch physician 7).

This sense of responsibility was linked to relieving the patient's suffering and not engaging in practices they saw as futile. Dutch physicians voiced a role as the filter between family wishes and the treatment decision, where family wishes were considered but the physician's decision ultimately prevailed.

"If I have the feeling that I'm not only prolonging suffering but also making suffering worse, then I will be very strong and say 'no'" (Dutch physician 12).

In contrast, American physicians were more likely to report their role as one that provided information to the family in a passive and deferential fashion.

"If I had my druthers, I suppose I would transport very, very few patients out of the nursing home. But it's really not my decision. I'm there as a medical provider, and these decisions about how much care each individual patient is going to receive is really made by the family for these demented folks. I am there to provide information" (US physician 9).

\section{Family's Patient Care Wishes}

Family wishes, or the preferences regarding care, were important in both cultures, but American physicians were more deferential to the family, often citing the possibility of threat from the family.
"It's not appropriate to send the patient to the hospital to treat the pneumonia. But when push comes to shove, if I advise not doing it-let the patient just die in peace-and the family says, 'No, we want them to go to the hospital,' they go to the hospital because I don't want to be sued" (US physician 4).

Although a few Dutch physicians mentioned a concern for disciplinary action that families could potentially take against them, their inclination to do what was best for the patient superseded those fears.

"She was very demented, could not drink or eat, didn't know anything, and was not conscious. I didn't want to transport her to the hospital anymore, and despite what the family wanted, that was my decision. That is a decision that you sometimes have to make, as a doctor" (Dutch physician 3).

\section{Process of Physician Treatment Decision}

Physician treatment decisions resulted from a process that included an interpretation of the patient's course of illness, quality of life, functional status, and perceived resuscitation preferences, followed by negotiation with the family. American physicians described the patient's quality of life as often as Dutch physicians; however, they were less likely to reflect upon it in their decision making and more likely to acknowledge the discomfort they felt arriving at decisions they did not believe were appropriate:

"Many times I feel that instead of prolonging life, which I take very seriously, many times I feel like all I'm doing is postponing death" (US physician 4).

American physicians tried to inform families of their opinion regarding appropriate care but ultimately were deferential, letting families direct the course of care they wanted, despite the physician's preferences.

"You need to understand if we do go back to the hospital, we're going to unleash a medical evaluation apparatus that means multiple testing, and it's hard to control that. I tell them I'm not sure this is the thing that's best for your loved one. I try to go through it and then I usually defer" (US physician 11).

Although Dutch physicians kept family members involved in the decision-making process and helped families grieve and cope, their sense of responsibility to the patient was primary. As a result, the process was physician driven.

"I am used to really taking time and effort, really trying to connect to the family member that is trying to do his or her best for our patient ... by investing time and really trying to connect with someone, really telling what my viewpoint is. And in my experience that almost every time leads to the fact that you all together come to the right decision" (Dutch physician 6).

"It is a matter of trying to keep everyone satisfied, 
as though you are in a rocking boat and trying to maneuver through the whole process, and everyone at the end of the process has the feeling that that was a nice piece of maneuvering and has a good feeling about it" (Dutch physician 2).

In their process of decision making, Dutch physicians reported placing a high value on patient quality of life and used that information as a key determinant as to whether a patient was treated aggressively. Dutch nursing home physicians often cited the ways in which they acquired their close knowledge of patients and their families, which was helpful when an acute illness arose.

"Because I look after patients during months usually, and during years often, I know them very well. Not only the patients, but also the relatives. So when there is a crisis, we have been through quite a bit before we arrived at this point where we are going to have this ultimate talk" (Dutch physician 4).

Although American physicians also acknowledged that previous and ongoing care discussions would be ideal, they often broached them only during an acute illness.

"You'll improve the odds of not being inappropriately aggressive if you talk with the family ahead of time. If you wait until the patient is acutely ill and then ask them to consider just letting the patient die peacefully, generally they can not think straight at that time and will opt for the more aggressive treatment" (US physician 4).

Where Dutch physicians used quality-of-life assessment, American physicians cited the patient's code status as a major consideration in the process of making treatment decisions, expressing frustration at the obligation to transport patients from the nursing home to the hospital if the patient did not have a "no code" or "do not resuscitate" order.

"The 'full code' ones are a little more difficult, because we probably already failed them at some point along the way in that they shouldn't be a full code, so there's been a problem some place. Either the family doesn't really understand, or I haven't really done an adequate job of explaining the difference between code and no code. But given that, if they're a full code and they're obviously becoming sick and something acute is happening, then occasionally we can keep them, but frequently we have to transport them out" (US physician 9).

In Dutch nursing homes, patients with dementia are not candidates for resuscitation, which is considered inappropriate and futile, and most Dutch physicians do not even discuss the possibility with families.

"I do not offer resuscitation when I talk to relatives or to a patient, because I think it's a silly notion, when you talk about demented patients. The outcome is a much worse patient and the suffering will increase many fold. So we don't offer that as an option" (Dutch physician 4).
There were differences within the 2 groups of physicians, as well as similarities between the US and Dutch physicians. US physicians also described cases for which treatment was withheld, and Dutch physicians were sometimes directive in recommending treatment.

"She was very demented and generally unhappy. We can't cure her of dementia, and she is never going to get better. We could have saved her to die again in a few months or weeks. I look at it as the natural progression of the disease, and feeding tubes and antibiotics just are not appropriate. To me, helping someone pass away in a comfortable way is rewarding and you feel very protective. It is important to change gears from helping them to live to helping them to die nicely" (US physician 10).

"She was acutely ill with pneumonia. But she was, before she became ill, in a quite good condition, although the dementia was quite severe. I didn't discuss it with the family. I told them she had pneumonia and that she has to be treated. I think I treat everyone unless there is a reason the treatment would not be successful" (Dutch physician 7).

\section{DISCUSSION}

Although physicians in the United States and in the Netherlands who care for demented elders in longterm care settings work in different social and cultural environments, both shared the belief that nonaggressive care was sometimes appropriate when demented nursing home patients became ill with pneumonia. These individual beliefs, however, were manifested differently for US and Dutch physicians in their role perceptions, in the process of their decision making, and in the way they responded to the patients' family's wishes.

The discordance between individual beliefs and social roles points to the importance of contextual elements within the physicians' environment. To account graphically for these influences on physician decision making, we constructed conceptual models for US and Dutch physicians (Figures 1 and 2). Both models embed 3 contextual elements_-societal values and constraints, technological medical training and values, and the policies and practices of the nursing home-as background factors that affect influences on physician decision making.

Figure 1 depicts contextual elements and influences on US physician decision making. The dominant contextual element in this model is technological medical training and values. American nursing homes are generally staffed by physicians trained in internal medicine and family medicine, where acute-care hospitals are primary training sites, and emphasis is placed on technology and diagnostics. ${ }^{14,15}$ The organization of nursing home care in the United States further constrains and shapes the 


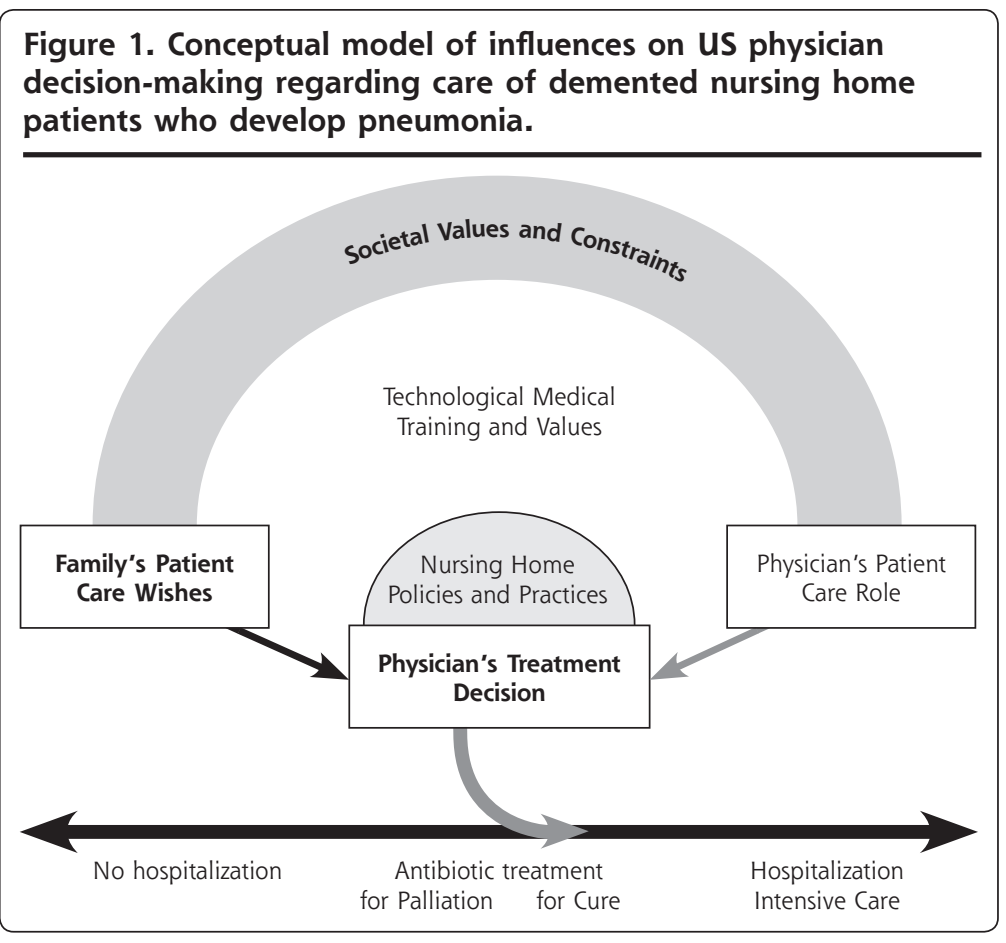

Figure 2. Conceptual model of influences on Dutch physician decision-making regarding care of demented nursing home patients who develop pneumonia.

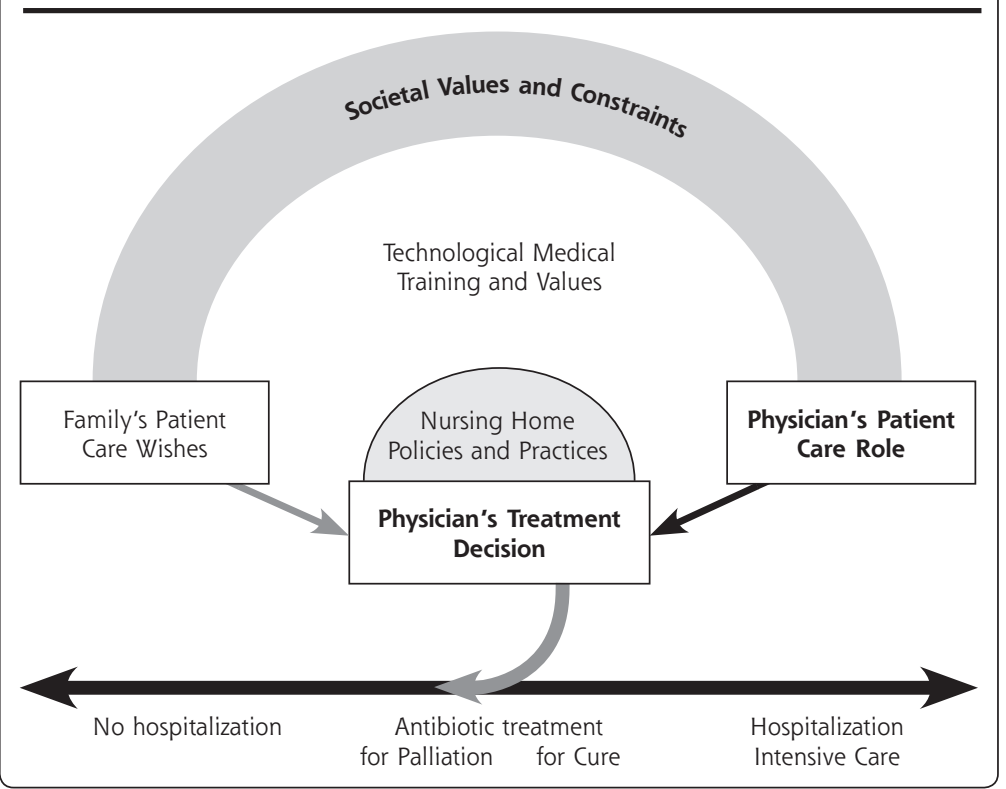

The organization of nursing home care also leads to a reduced sense of physician responsibility and a system in which medical decision making is often deferred to others, such as the family or hospital physicians. Larger societal values, such as personal autonomy, and constraints, such as litigation, play a part as well. Compared with their Dutch counterparts, US physicians perceived more threat from the family, most likely because they do not know the family well, and perhaps because they also work in a more uncertain legal and ethical climate. ${ }^{17}$ These contextual elements result in American physicians seeing their roles as more passive and neutral, diminishing their influence in the treatment decision and placing higher value on the perceived wishes of the family, and resulting in deferral to more-aggressive care along the treatment spectrum (Figure 1).

For Dutch physicians, the societal values and constraints include the wide public discussion regarding end-of-life care and the place of suffering in the Netherlands. ${ }^{17,18}$ Physician training of nursing home physicians, a second contextual element, places less emphasis on technology than in the United States. In the Netherlands, nursing home medicine is a specialty for which physicians are trained exclusively in nursing home settings and then employed there as their only site of practice. ${ }^{19}$ This organization of care provides the venue for physicians to get to know their patients and patients' families; discussions can be facilitated and are more likely to occur, even before an acute illness. Although family input is important, Dutch physicians are more active in directing the family, embodying both societal and medical values that expect them to assume primary responsibility. As a result of their attitude in acting in the physician's role, as nursing home responsibilities are frequently a small part of a physician's overall clinical practice, and busy physicians spend limited time there. ${ }^{16}$ In our study, American physicians frequently mentioned the lack of time for seeing patients at nursing homes, citing the poor financial reimbursement they receive for such work and their need to spend time at their primary site of practice, which is usually an office or hospital setting. best interest of the patient, physician decision making favors the less-aggressive end of the treatment spectrum in our model (Figure 2). We included antibiotics for palliation in the model, however, as antibiotic treatment is a practice of Dutch physicians, ${ }^{4}$ even though evidence of the palliative effects of antibiotics is lacking. ${ }^{20}$

We triangulated our conceptual models with the perspective of society and the family, both of which play an 
important role in our model. Triangulation uses multiple data sources to support and enhance the understanding of the phenomenon under study. ${ }^{21,22}$ To begin, standards of care, although they are advanced by the health care profession, ultimately require the endorsement of society. ${ }^{23}$ Our model depicting the influence of Dutch society on physician decision making is supported by Dutch law and various Dutch medical professional organizations, which explicitly recognize that treatment may be withheld if the physician considers treatment futile, with the single Dutch guideline issued on the topic focusing on when to withhold antibiotics. ${ }^{24}$ In contrast, treatment guidelines for nursing home-acquired pneumonia in the United States focus on decisions related to hospitalization, choice of antibiotics, and when to switch from parenteral to oral treatment. ${ }^{25}$ Cure of the acute disease is often the primary focus, even with an underlying diagnosis of severe dementia. ${ }^{2}$

The Netherlands has even taken the controversial step of decriminalizing physician euthanasia, if certain procedures are followed and specific requirements are met, including the explicit request of a competent patient. The requirement for competency limits the practice of euthanasia in patients with dementia, and physician-assisted suicide in nursing home settings is rare, even in patients with an advance euthanasia directive. ${ }^{26,27}$ The Netherlands, however, has recently issued strict guidelines for the deliberate ending of life for newborns who live with severe and sustained suffering from incurable conditions, evidence that they do believe proxy decision makers can make end-of-life decisions for incompetent patients. ${ }^{28}$ Physician-assisted suicide, as embodied in the Oregon right-to-death movement, remains a contested ethical and legal issue in the United States, with many initiatives challenged in the court system. Although our study did not address euthanasia, this background highlights the societal differences between the United States and the Netherlands regarding attitudes toward death and dying. From an ethical point of view, active euthanasia is distinct from actions intended to hasten the end of life by withholding curative care and from administering medications primarily to manage pain or other symptoms but with the potential for hastening death. ${ }^{29}$

Families play an important role in our models, and physicians frequently mentioned their role in shared decision making. Family members in the United States continue to report dissatisfaction with the quality of end of-life care delivered to their family member who died in a nursing home setting, citing high rates of unmet needs for symptom management, concerns about physician communication regarding medical decision making, a lack of emotional support for themselves, and a belief that their dying family member was not always treated with respect. ${ }^{30-32}$ Dutch physicians explicitly consider families' feelings and preferences, ${ }^{33}$ but Dutch families' attitudes and experiences need to be studied in greater detail.

Our findings suggest that Dutch physicians are more directive in their communications, exhibiting paternalism toward patients and their families. In contrast, physicians in the United States have moved away from this approach and toward a greater emphasis on patient autonomy. ${ }^{34}$ One risk of patient autonomy, however, is the withholding of physician clinical experience and recommendations regarding care. Beyond patient autonomy, physicians have the duty of beneficence to share their judgments about the futility or success of interventions or treatments. ${ }^{35}$ Quill and Brody have proposed an "enhanced autonomy" model that encourages patients, surrogate decision makers, and physicians to actively exchange ideas, explicitly negotiate differences, and share power and influence to serve the patient's best interests, recommendations that arise from this process promote a collaboration among patient, families, and the physician so that choices are informed by both the medical facts and the physician's experience. ${ }^{36}$ The current structure of nursing home care in the United States inhibits the implementation of this model, because it requires regular communication and a relationship with the patient and the family. In contrast, Dutch nursing home physicians have the structure to develop these relationships and utilize this model but would need to consider a less paternalistic approach to their care.

There are several limitations to our study. As an exploratory study, the conceptual model should be considered preliminary and open to modification. Qualitative studies are not intended to be statistically representative of any population but to provide an in-depth examination of complex phenomena. The frequency and validation of participating physicians' experiences were not determined. There were many similarities between the US and Dutch physicians, as well as differences within the 2 groups, but the contextual influences were strong enough to emphasize the differences between the groups. Because we were interested in the physicians' viewpoint, we did not quantify the degree of dementia or pneumonia in the patients. There are more nursing home beds per capita in the United States than in the Netherlands, so it could be that Dutch nursing home patients are sicker. ${ }^{37}$ Even so, the strength of this investigation lies in the in-depth examination and the emerging conceptual model, which depicts a complex interplay of medical and nursing home systems and societal phenomena. Finally, our results and model are based on the physician's perspective and do not address the patient, family, or other nursing home employee view.

In summary, we found that physicians from the 
United States and the Netherlands described differences in their patient care roles, the family's patient care wishes, and the process by which they make treatment decisions regarding care of demented nursing home patients who develop pneumonia. Efforts to improve the care of these patients must involve consideration of the context of societal values, the location of physician training, and the processes by which physicians determine and negotiate patient and family preferences regarding care.

\section{To read or post commentaries in response to this article, see it} online at http://www.annfammed.org/cgi/content/full/4/3/221.

Key words: Dementia; nursing home medicine; pneumonia; palliative care; end of life care; euthanasia, passive

Submitted July 25, 2005; submitted, revised, November 11, 2005; accepted November 21, 2005.

Funding support: The US National Institute on Aging (Grant \#AG01033), the Fetzer Institute, and the Donald W. Reynolds Foundation provided funding support. The authors had full access to all of the data in the study and take responsibility for the integrity of the data and the accuracy of the data analysis.

\section{References}

1. Anderson RN, Smith BL. Deaths: leading causes for 2001. Natl Vital Stat Rep. 2003;52:1-85.

2. Morrison RS, Siu AL. Survival in end-stage dementia following acute illness. JAMA. 2000;284:47-52.

3. Riesenberg D. Hospital care of patients with dementia. JAMA. 2000;284:87-89.

4. van der Steen JT, Ooms ME, Ader HJ, Ribbe MW, van der Wal G. Withholding antibiotic treatment in pneumonia patients with dementia: a quantitative observational study. Arch Intern Med. 2002; 162:1753-1760.

5. Fried TR, Gillick MR, Lipsitz LA. Whether to transfer? Factors associ ated with hospitalization and outcome of elderly long-term care patients with pneumonia. J Gen Intern Med. 1995;10:246-250.

6. Muder RR. Pneumonia in residents of long-term care facilities: epidemiology, etiology, management, and prevention. Am J Med. 1998;105:319-330.

7. van der Steen JT, Kruse RL, Ooms ME, et al. Treatment of nursing home residents with dementia and lower respiratory tract infection in the United States and The Netherlands: an ocean apart. J Am Geriatr Soc. 2004;52:691-699.

8. Mehr DR, van der Steen JT, Kruse RL, et al. Lower respiratory infections in nursing home residents with dementia: a tale of two countries. Gerontologist. 2003;43 Spec No 2:85-93.

9. Kruse RL, Mehr DR, Boles KE, et al. Does hospitalization impact survival after lower respiratory infection in nursing home residents? Med Care. 2004;42:860-870.

10. Boockvar KS, Gruber-Baldini AL, Burton L, et al. Outcomes of infection in nursing home residents with and without early hospital transfer. J Am Geriatr Soc. 2005;53:590-596.

11. Forbes S, Bern-Klug M, Gessert C. End-of-life decision making for nursing home residents with dementia. J Nurs Scholarsh. 2000;32:251-258.

12. Crabtree BF, Miller WL. Doing Qualitative Research. 2nd ed. Thousand Oaks, Calif: Sage Publications; 1999.

13. QSR N6. Version Melbourne, Australia: QSR International Pty Ltd; 2002.

14. Accreditation Council for Graduate Medical Education (ACGME). Program requirements for residency education in family medicine. 2001. Available at: http://www.acgme.org.
15. Accreditation Council for Graduate Medical Education (ACGME). Program requirements for residency education in internal medicine. 2003. Available at: http://www.acgme.org.

16. McCartney RD, McCartney BJ. Nursing home visits: an efficient system for the busy physician. Geriatrics. 1997;52:57-60, 65, 69.

17. Quill TE, Kimsma G. End-of-life care in The Netherlands and the United States: a comparison of values, justifications, and practices. Camb Q Healthc Ethics. 1997;6:189-204.

18. Pijnenborg L. End-of-Life Decisions in Dutch Medical Practice. [Thesis] Rotterdam: Erasmus University, Department of Public Health; 1994.

19. Hoek JF, Ribbe MW, Hertogh CM, van der Vleuten CP. The specialist training program for nursing home physicians: a new professional challenge. J Am Med Dir Assoc. 2001;2:326-330.

20. van der Steen JT, Ooms ME, van der Wal G, Ribbe MW. Pneumonia: the demented patient's best friend? Discomfort after starting or with holding antibiotic treatment. J Am Geriatr Soc. 2002;50:1681-1688.

21. Miles M, Huberman A. Qualitative Data Analysis. 2nd ed. Thousand Oaks, Calif: Sage Publications; 1994.

22. Rice PL, Ezzy D. Qualitative Research Methods. Cambridge: Oxford University Press; 2001.

23. Schneiderman LJ, Jecker N. Futility in practice. Arch Intern Med. 1993; 153:437-441.

24. van der Steen JT, Muller MT, Ooms ME, van der Wal G, Ribbe MW. Decisions to treat or not to treat pneumonia in demented psychogeriatric nursing home patients: development of a guideline. J Med Ethics. 2000;26:114-120.

25. Naughton BJ, Mylotte JM. Treatment guideline for nursing home acquired pneumonia based on community practice. J Am Geriatr Soc. 2000;48:82-88

26. Rurup ML, Onwuteaka-Philipsen BD, van der Heide A, van der Wal $G$, van der Maas PJ. Physicians' experiences with demented patients with advance euthanasia directives in the Netherlands. J Am Geriatr Soc. 2005;53:1138-1144.

27. van der Steen JT, van der Wal G, Mehr DR, Ooms ME, Ribbe MW. End-of-life decision making in nursing home residents with dementia and pneumonia: Dutch physicians' intentions regarding hastening death. Alzheimer Dis Assoc Disord. 2005;19:148-155.

28. Verhagen AA, Sauer PJ. End-of-life decisions in newborns: an approach from The Netherlands. Pediatrics. 2005;116:736-739.

29. Quill TE, Lee BC, Nunn S. Palliative treatments of last resort: choosing the least harmful alternative. University of Pennsylvania Center for Bioethics Assisted Suicide Consensus Panel. Ann Intern Med. 2000;132:488-493.

30. Teno JM, Clarridge BR, Casey V, et al. Family perspectives on endof-life care at the last place of care. JAMA. 2004;291:88-93.

31. Hanson LC, Danis M, Garrett J. What is wrong with end-of-life care? Opinions of bereaved family members. J Am Geriatr Soc 1997;45:1339-1344.

32. Shield RR, Wetle T, Teno J, Miller SC, Welch L. Physicians "missing in action": family perspectives on physician and staffing problems in end-of-life care in the nursing home. J Am Geriatr Soc. 2005;53:1651-1657.

33. The AM, Pasman R, Onwuteaka-Philipsen B, Ribbe M, van der Wal G. Withholding the artificial administration of fluids and food from elderly patients with dementia: ethnographic study. BMJ. 2002;325:1326.

34. Laine C, Davidoff F. Patient-centered medicine. A professional evolution. JAMA. 1996;275:152-156.

35. Schneiderman LJ, Jecker NS, Jonsen AR. Medical futility: response to critiques. Ann Intern Med. 1996;125:669-674.

36. Quill TE, Brody H. Physician recommendations and patient autonomy: finding a balance between physician power and patient choice Ann Intern Med. 1996;125:763-769.

37. Ribbe MW, Ljunggren G, Steel K, et al. Nursing homes in 10 nations: a comparison between countries and settings. Age Ageing. 1997;26 Suppl 2:3-12. 